Yukawa Institute

YITP-96-49

Kyoto University

KUNS-1415

\title{
The Minimum Total Mass of MACHOs and Halo Models of the Galaxy
}

\author{
T. Nakamura \\ Yukawa Institute for Theoretical Physics, Kyoto University, Kyoto 606 \\ and \\ Y. Kan-ya and R. Nishi \\ Department of Physics, Kyoto University, Kyoto 606
}

\begin{abstract}
If the density distribution $\rho(r)$ of MACHOs is spherically symmetric with respect to the Galactic center, it is shown that the minimal total mass $M_{m i n}^{\mathrm{MACHO}}$ of the MACHOs is $1.7 \times 10^{10} M_{\odot} \tau_{-6.7}^{\mathrm{LMC}}$ where $\tau_{-6.7}^{\mathrm{LMC}}$ is the optical depth $\left(\tau^{\mathrm{LMC}}\right)$ toward the Large Magellanic Cloud (LMC) in the unit of $2 \times 10^{-7}$. If $\rho(r)$ is a decreasing function of $r$, it is proved that $M_{\text {min }}^{\mathrm{MACHO}}$ is $5.6 \times 10^{10} M_{\odot} \tau_{-6.7}^{\mathrm{LMC}}$. Several spherical and axially symmetric halo models of the Galaxy with a few free parameters are also considered. It is found that $M_{m i n}^{\mathrm{MACHO}}$ ranges from $5.6 \times 10^{10} M_{\odot} \tau_{-6.7}^{\mathrm{LMC}}$ to $\sim 3 \times 10^{11} M_{\odot} \tau_{-6.7}^{\mathrm{LMC}}$. For general case, the minimal column density $\Sigma_{m i n}^{\mathrm{MACHO}}$ of MACHOs is obtained as $\Sigma_{m i n}^{\mathrm{MACHO}}=25 \mathrm{M}_{\odot} \mathrm{pc}^{-2} \tau_{-6.7}^{\mathrm{LMC}}$. If the clump of MACHOs exist only halfway between LMC and the sun, $M_{\text {min }}^{\mathrm{MACHO}}$ is $1.5 \times$ $10^{9} M_{\odot}$. This shows that the total mass of MACHOs is smaller than $5 \times 10^{10} M_{\odot}$, i.e. $\sim$ $10 \%$ of the mass of the halo inside LMC, either if the density distribution of MACHOs is unusual or $\tau^{\mathrm{LMC}} \ll 2 \times 10^{-7}$.

(11 October 1996, Accepted for publication in Apj. Letters.)
\end{abstract}

Subject headings: dark matter — Galaxy: halo — Galaxy: structure — gravitational lensing 


\section{Introduction}

Recent second year analysis of microlensing events toward the Large Magellanic Cloud (LMC) by the MACHO collaboration suggests that the optical depth $\tau^{\mathrm{LMC}}$ is $\sim 2 \times 10^{-7}$ and the fraction $f$ of MACHOs is $\sim 0.4$ with the typical mass $\sim 0.34 M_{\odot}$ in the standard spherical flat rotation halo model Bennett et al. 1996). The estimated mass of MACHOs is just the mass of red dwarfs. However the contribution of the halo red dwarfs to MACHO events should be small since the observed density of the halo red dwarfs is too low Bahcall et al. 1994, Graff \& Freese 1996a, Graff \& Freese 1996b).

As for the white dwarf Galaxy halo, Charlot \& Silk (1995) combined population synthesis models with constraints from deep galaxy surveys and showed that only a small fraction $(\leq 10 \%)$ of the dark mass in the present-day galaxy halo could be in the form of white dwarf remnants (WDR) of intermediate-mass stars. Adams \& Laughlin (1996) recently argued the implications of white dwarf Galactic halos. From the current limits on the density of red dwarfs Bahcall et al. 1994, Graff \& Freese 1996a, Graff \& Freese 1996b) and the galactic metallicity, the IMF must be sharply peaked about a characteristic mass scale $M_{c} \sim 2.3 M_{\odot}$. They concluded that the mass fraction of WDRs in the halo is likely to be less than $25 \%$ since only a fraction of the initial mass of a star is incorporated into WDRs.

The spatial density distribution function of MACHOs which caused microlensing events is not known in spite of many arguments on the mass distribution of halo dark matter Paczyński 1986. Griest 1991, Sackett \& Gould 1993, Frieman \& Scoccimarro 1994, Sahu 1994. Alcock et al. 1995. Gates, Gruk \& Iurner 1996. Turner, Gates, \& Gyuk 1996, Evans 1996, Kan-ya, Nishi \& Nakamura 1996). Only the possible value of the optical depth $\tau^{\text {LMC }}$ Bennett et al. 1996) is known, the fraction of MACHOs in the halo depending on the spatial density distribution function of MACHOs. In this situation it is important to check the relation between the total mass $M^{\mathrm{MACHO}}$ of MACHOs to the density distribution function of MACHOs for the given optical depth $\tau^{\mathrm{LMC}}$. The results of such a study will be useful in the arguments on the fraction of MACHOs in the halo and what MACHOs are.

In this paper we study $M^{\mathrm{MACHO}}$ for various density distribution functions of MACHOs and discuss the minimal total mass of the MACHO halo. Gates, Gyuk, and Turner (1996) and Turner, Gates, and Gyuk (1996) also discussed the mass of the MACHO halo for various galaxy models. While they separately added unidentified dark thick disk components, we discuss the total mass responsible for microlensing. In $\S 2$ we obtain the minimal total mass $M_{m i n}^{\mathrm{MACHO}}$ of MACHOs for spherically symmetric density distribu- tions. In $\S 3$ we discuss $M_{m i n}^{\mathrm{MACHO}}$ for various axially symmetric density distributions. $\S 4$ will be devoted to discussions.

\section{Spherically Symmetric Halo Models and the Minimal Total Mass of MACHOs}

We assume that the density distribution function $\rho(r)$ of MACHOs is a function of the galactocentric radius $r$. The optical depth $\tau^{\text {LMC }}$ toward LMC is given by (Paczyński 1986, Griest 1991)

$$
\begin{gathered}
\tau^{\mathrm{LMC}}=\frac{4 \pi G}{c^{2}} \int_{0}^{D_{s}} x\left(1-\frac{x}{D_{s}}\right) \rho(r) d x, \\
r^{2}=R_{0}^{2}-2 R_{0} \eta x+x^{2},
\end{gathered}
$$

and

$$
\eta=\cos b \cos l,
$$

where $D_{s}, l, b$ and $R_{0}$ are the distance to LMC (50kpc), the galactic longitude and latitude of LMC and the galactocentric radius of the sun $(8.5 \mathrm{kpc})$, respectively. In Eq. (1) we assumed the threshold $u_{T}=1$ for simplicity.

Equation (1) is rewritten as

$$
\begin{gathered}
\tau^{\mathrm{LMC}}=\int f(x) d m, \\
f(x)=\frac{G}{c^{2}} \frac{x\left(1-\frac{x}{D_{s}}\right)}{r^{2} \frac{d r}{d x}},
\end{gathered}
$$

and

$$
d m=4 \pi r^{2} \rho(r) \frac{d r}{d x} d x .
$$

For LMC, $f(x)$ is infinite at $x=x_{c} \equiv R_{0} \eta=0.153 R_{0}$ so that the minimal total mass $M_{m i n}^{\mathrm{MACHO}}$ of MACHOs is zero for any given $\tau^{\mathrm{LMC}}$ if MACHOs are distributed in an infinitesimally thin shell at $r=r_{c} \equiv$ $\sqrt{1-\eta^{2}} R_{0}$. However this is wrong. Since the angular size of $\mathrm{LMC}$ is $\sim 10^{\circ} \times 10^{\circ}, M^{\mathrm{MACHO}}$ is minimized if MACHOs are distributed in a shell at $r=r_{c}$ with width $d$ given by

$$
d=\frac{10 \pi}{180} R_{0} \eta=227 \mathrm{pc} .
$$

It is easy to show that $M_{\text {min }}^{\mathrm{MACHO}}$ is given by

$$
\begin{gathered}
M_{\text {min }}^{\mathrm{MACHO}}=\frac{c^{2} \tau^{\mathrm{LMC}}}{3 G} \frac{\left(r_{c}+d\right)^{3}-r_{c}^{3}}{2 \sqrt{2 r_{c} d+d^{2}}\left(R_{0} \eta-\frac{R_{0}^{2} \eta^{2}+6 r_{c} d+3 d^{2}}{3 D_{s}}\right)} \\
=1.7 \times 10^{10} M_{\odot} \tau_{-6.7}^{\mathrm{LMC}},
\end{gathered}
$$

where $\tau_{-6.7}^{\mathrm{LMC}}$ is $\tau^{\mathrm{LMC}}$ in the unit of $2 \times 10^{-7}$. This shows that in principle $M^{\mathrm{MACHO}}$ can be only $\sim 3 \%$ 
of the total mass of the halo inside LMC. However the density distribution function of MACHOs in this case is very peculiar so that we calculate $M^{\mathrm{MACHO}}$ for more realistic $\rho(r)$ to know more realistic $M_{m i n}^{\mathrm{MACHO}}$. We consider two models;

\section{1)Polytropic Model} $R_{p}$.

$\rho(r)$ is given by polytrope of index $\mathrm{N}$ and the radius

2) $\alpha$ Model

$\rho(r)$ is given by

$$
\rho(r)=\frac{\rho_{0}}{\left(1+\frac{r^{2}}{R_{a}^{2}}\right)^{\alpha}} .
$$

This model is similar to the beta model of the cluster of galaxies with core radius $R_{a}$.

In figure 1 we show $M^{\mathrm{MACHO}}$ as a function of $R_{p}$ for several polytropic indices N. $M_{m i n}^{\mathrm{MACHO}}$ ranges from $5.6 \times 10^{10} M_{\odot} \tau_{-6.7}^{\mathrm{LMC}}$ for $\mathrm{N}=0$ to $7.8 \times 10^{10} M_{\odot} \tau_{-6.7}^{\mathrm{LMC}}$ for $\mathrm{N}=3$. For $\mathrm{N}=4$ and $4.5, M^{\mathrm{MACHO}}$ is greater than $1.0 \times 10^{11} M_{\odot} \tau_{-6.7}^{\mathrm{LMC}}$ and the minimum does not exist for $R_{p}<D_{s}$. Under the assumption that $\rho(r)$ is a decreasing function of $r$, it is shown in the Appendix that $M^{\mathrm{MACHO}}$ is minimized when $\rho(r)$ is constant. Therefore $M_{m i n}^{\mathrm{MACHO}}$ is $5.6 \times 10^{10} M_{\odot} \tau_{-6.7}^{\mathrm{LMC}}$ if $\rho(r)$ is a decreasing function. In figure 2 we show the total mass of MACHOs inside LMC in $\alpha$ models as a function of $R_{a}$ for several values of $\alpha$. $M_{m i n}^{\mathrm{MACHO}}$ ranges from $1.3 \times 10^{11} M_{\odot} \tau_{-6.7}^{\mathrm{LMC}}$ for $\alpha=1.5$ to $8.7 \times 10^{10} M_{\odot} \tau_{-6.7}^{\mathrm{LMC}}$ for $\alpha=6$. For large $\alpha, M_{\text {min }}^{\mathrm{MACHO}}$ does not change so much and it converges although the value of $R_{a}$ at the minimum increases . This behavior can be understood analytically using the asymptotic expression of gamma functions.

\section{Axially Symmetric Halo Models and the Minimal Total Mass of the MACHOs}

There are several suggestions that the Galactic halo is not spherically symmetric Aarseth \& Binney 1978, Aguilar \& Merritt 1990, Binney 1994) so that we study here axially symmetric halo models and calculate $M^{\mathrm{MACHO}}$. We consider two models;

1) Exponential Disk Model

The axially symmetric density distribution function $\rho(R, Z)$ in cylindrical coordinates is given by

$$
\rho(R, Z)=\rho_{0} \exp \left(-\frac{R}{R_{d}}-\frac{|Z|}{Z_{d}}\right),
$$

where $R_{d}$ and $Z_{h}$ are scale heights.

2) Elliptical Model

$\rho(R, Z)$ is given by

$$
\rho(R, Z)=\frac{\rho_{0}}{\left(1+\frac{R^{2}}{a^{2}}+\frac{Z^{2}}{c^{2}}\right)^{\alpha}},
$$

where $a$ and $c$ describe the ellipticity of the equidensity surface. This is an axially symmetric version of the $\alpha$ model.

In figure 3 we show $M^{\mathrm{MACHO}}$ as a function of $R_{d}$ in exponential disk models for various aspect ratios $\left(Z_{h} / R_{d}\right)$ of the equidensity surface. We see $M_{\text {min }}^{\mathrm{MACHO}}$ is $\sim 1.0 \times 10^{11} M_{\odot} \tau_{-6.7}^{\mathrm{LMC}}$ for $0.5<Z_{h} / R_{d}<1.0$ and it increases with the decrease of $Z_{h} / R_{d}$ for $Z_{h} / R_{d}<0.5$.

In figure $4 \mathrm{a}$ and $4 \mathrm{~b}$ we show $M^{\mathrm{MACHO}}$ in elliptical models as a function of $a$ and the aspect ratio $c / a$ for $\alpha=2.5$ and $\alpha=6.0$, respectively. $M_{\text {min }}^{\mathrm{MACHO}}$ is $8.9 \times 10^{10} M_{\odot} \tau_{-6.7}^{\mathrm{LMC}}$ at $a=10 \mathrm{kpc}$ and $c=6 \mathrm{kpc}$ for $\alpha=2.5$ and is $7.08 \times 10^{10} M_{\odot} \tau_{-6.7}^{\mathrm{LMC}}$ at $a=22 \mathrm{kpc}$ and $c=13.2 \mathrm{kpc}$ for $\alpha=6.0$. For large $\alpha, M_{\min }^{\mathrm{MACHO}}$ converges, similarly to the $\alpha$ models.

\section{Discussions}

A deep north Galactic pole proper motion survey( Majewski, Munn \& Hawley 1996) suggests that the halo is not dynamically mixed but contains a significant fraction of stars with membership in correlated stellar streams. If MACHOs are also dynamically unmixed, it is possible that the density distribution function is neither spherically nor axially symmetric but completely inhomogeneous. In such a case what we can say from the microlensing events toward LMC is the minimal column density $\Sigma_{\text {min }}^{\mathrm{MACHO}}$ of MACHOs. Since in Equation (1), $x\left(1-x / D_{s}\right)<D_{s} / 4, \Sigma_{\text {min }}^{\mathrm{MACHO}}$ is given by

$$
\Sigma_{\min }^{\mathrm{MACHO}}=25 M_{\odot} \mathrm{pc}^{-2} \tau_{-6.7}^{\mathrm{LMC}} .
$$

Similar to Equation (7), the linear size of the clump of MACHOs should be larger than $174 \mathrm{pc}(x / \mathrm{kpc})$ where $x$ is the distance to the clump of MACHOs . For $x=D_{s} / 2, M_{\min }^{\mathrm{MACHO}}$ is $1.5 \times 10^{9} M_{\odot}$. If this is the case, the optical depth toward the Small Magellanic Cloud will be quite different and the inhomogeneity of the density distribution of MACHOs can be checked.

In conclusion, it is shown that the total mass of MACHOs becomes smaller than $5 \times 10^{10} M_{\odot}$, i.e. $\sim$ $10 \%$ of the mass of the halo inside the LMC, either if the density distribution of MACHOs is unusual or $\tau^{\mathrm{LMC}} \ll 2 \times 10^{-7}$.

We like to thank Hayward for checking English. This work was supported by Grant-in-Aid of Scientific Research of the Ministry of Education, Culture, and Sports, No.07640399.

\section{Appendix}

The proof that $M^{\mathrm{MACHO}}$ is minimized if the spherically symmetric density distribution function $\rho(r)$ is a constant.

We first fix the density at $r=r_{c}$. Since the matter inside $r_{c}$ does not contribute to $\tau, M^{\mathrm{MACHO}}$ is mini- 
mized if $\rho(r)$ is constant for $r<r_{c}$. For $r \geq r_{c}$ from Equation (4) it is easy to observe that $d \tau / d m$ is a decreasing function of $r$ irrespective of $\rho(r)$. Now for fixed $m$ (i.e. the same mass but different $r$ depending on the density distribution function), $r$ is smallest and $d \tau / d m$ is largest if $\rho(r)=\rho\left(r_{c}\right)=$ constant. This means that $\tau$ is largest for constant density distribution for fixed total mass $M^{\mathrm{MACHO}}$. Inversely for fixed $\tau, M^{\mathrm{MACHO}}$ is minimized for the constant density distribution. By varying the value $\rho\left(r_{c}\right), M_{m i n}^{\mathrm{MACHO}}$ is obtained.

\section{REFERENCES}

Aarseth, S. J., \& Binney, J. J. 1978, MNRAS, 185,227

Adams, F.C. \& Laughlin, G. 1996 submitted to ApJ

Aguilar, L. A., \& Merritt, D. R. 1990, ApJ, 265, 33

Alcock, C. et al. 1995, ApJ, 449, 28

Ashman, K. M. 1992, PASP, 104, 1109

Bahcall, J. N., Flynn, C., Gould, A., \& Kirhakos, S. 1994, ApJ, 435, L51

Bennett, D. P. et al. 1996 , in Proc. 2nd International Workshop on Gravitational Microlensing Survey ed. M. Moniez

Binney, J. J. 1994, in IAU Symp. 169, Unsolved Problems of the Milky Way ed. by Blitz, L. (Dordrecht: Kluwer)

Charlot, S. \& Silk, J. 1995, ApJ, 445, 124

Evans, N.W. 1996, MNRAS, 278, L5

Fich, M., Blitz, L., \& Stark, A. 1989, ApJ, 342, 272

Fich, M., \& Tremaine, S. 1991, ARA\&A,29, 409

Flynn, C. \& Fuchs, B.. 1994 MNRAS, 270, 471

Frieman, J., \& Scoccimarro, R. 1994, ApJ, 431, L23

Gates, E., Gyuk, G. \& Turner, M.S., 1996, Phys. Rev. D, 53, 4138

Gould, A. 1990, MNRAS, 244, 25

Graff, D.S. \& Freese, K. 1996, ApJ, 456, L49

Graff, D.S. \& Freese, K. 1996, submitted to ApJ

Griest, K. 1991, ApJ, 366, 412

Kan-ya, Y. , Nishi, R. \& Nakamura, T. 1996, PASJ, 48,479

Majewski, S.R., Munn, J.A.\& Hawley, S.L. 1996, ApJ, 459, L73

Paczyński, B. 1986, ApJ, 304, 1
Sahu, K. C. 1994, Nature, 370, 275

Sackett, P. D., \& Gould, A. 1993, ApJ, 419, 648

Turner, M.S., Gates, E., \& Gyuk, G. 1996, astro$\mathrm{ph} / 9601168$

This 2-column preprint was prepared with the AAS LATEX macros $\mathrm{v} 4.0$ 
Fig. 1. - The total mass of MACHOs for the polytropic model in the unit of $M_{\odot} . \mathrm{N}$ is the polytropic index and $R_{p}$ is the radius of the density distribution of MACHOs.

Fig. 2.- The total mass of MACHOs for the $\alpha$ model in the unit of $M_{\odot}$. The density distribution is given by $\rho(r)=\frac{\rho_{0}}{\left(1+\frac{r^{2}}{R_{a}^{2}}\right)^{\alpha}}$.

Fig. 3.- The total mass of MACHOs for the exponential disk model in the unit of $M_{\odot}$. The density distribution is given by $\rho(R, Z)=\rho_{0} \exp \left(-\frac{R}{R_{d}}-\frac{|Z|}{Z_{d}}\right)$.

Fig. 4. - The total mass of MACHOs for the elliptic model in the unit of $M_{\odot}$. The density distribution is given by $\rho(R, Z)=\frac{\rho_{0}}{\left(1+\frac{R^{2}}{a^{2}}+\frac{Z^{2}}{c^{2}}\right)^{\alpha}}$. a) for $\alpha=2.5$ and b) for $\alpha=6.0$ 
The Total Mass of MACHOs for Polytropic Model (figure 1)

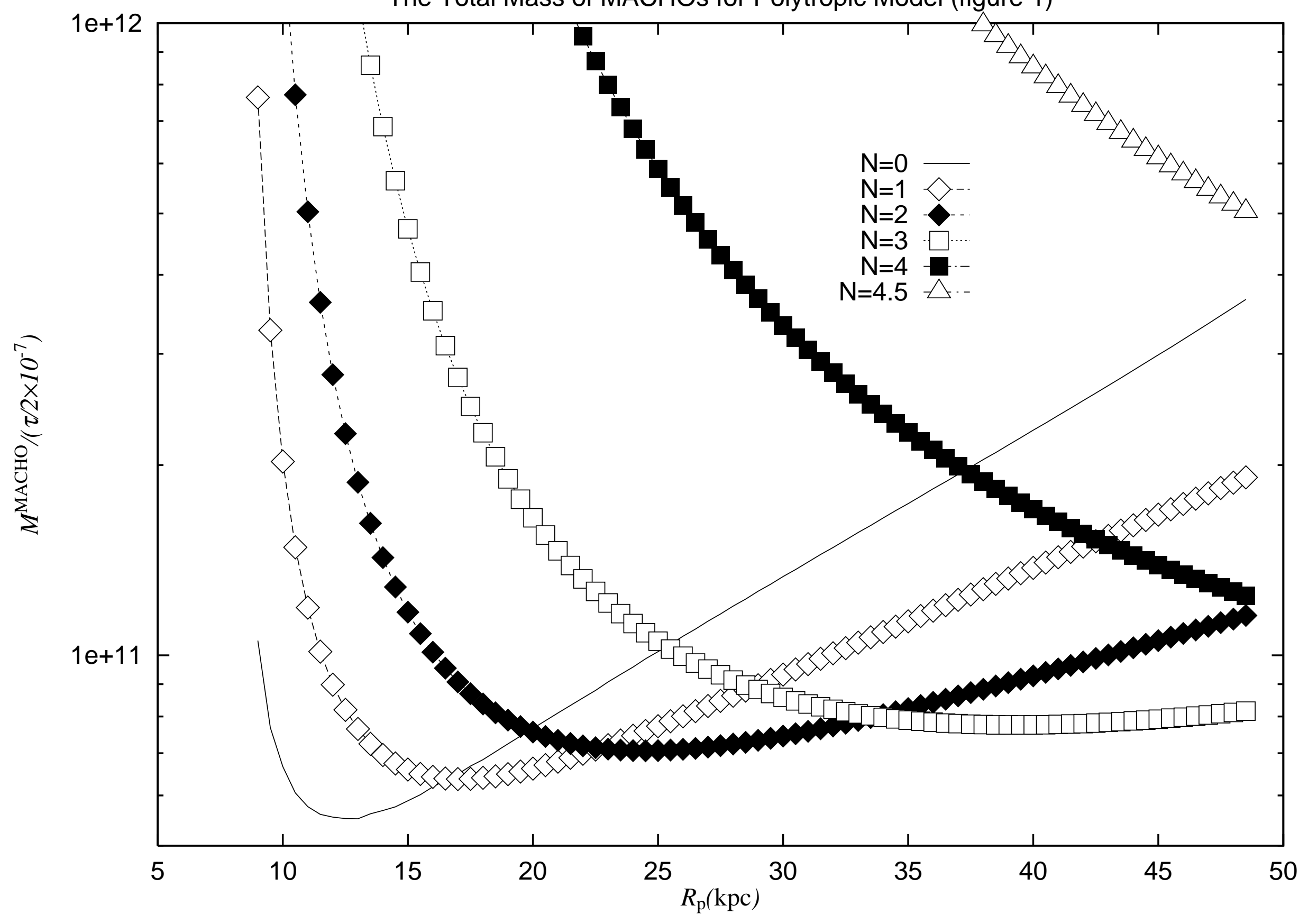




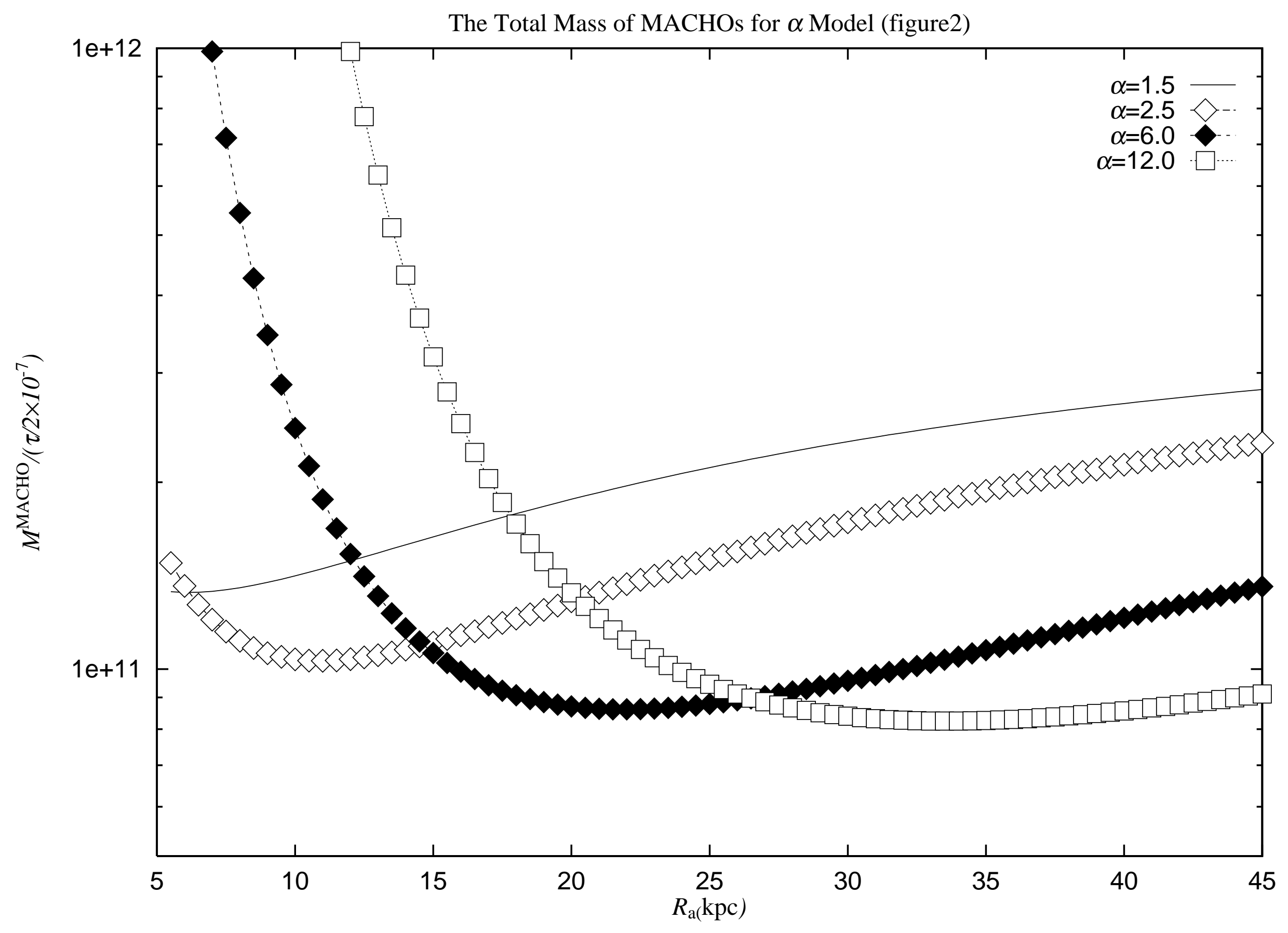




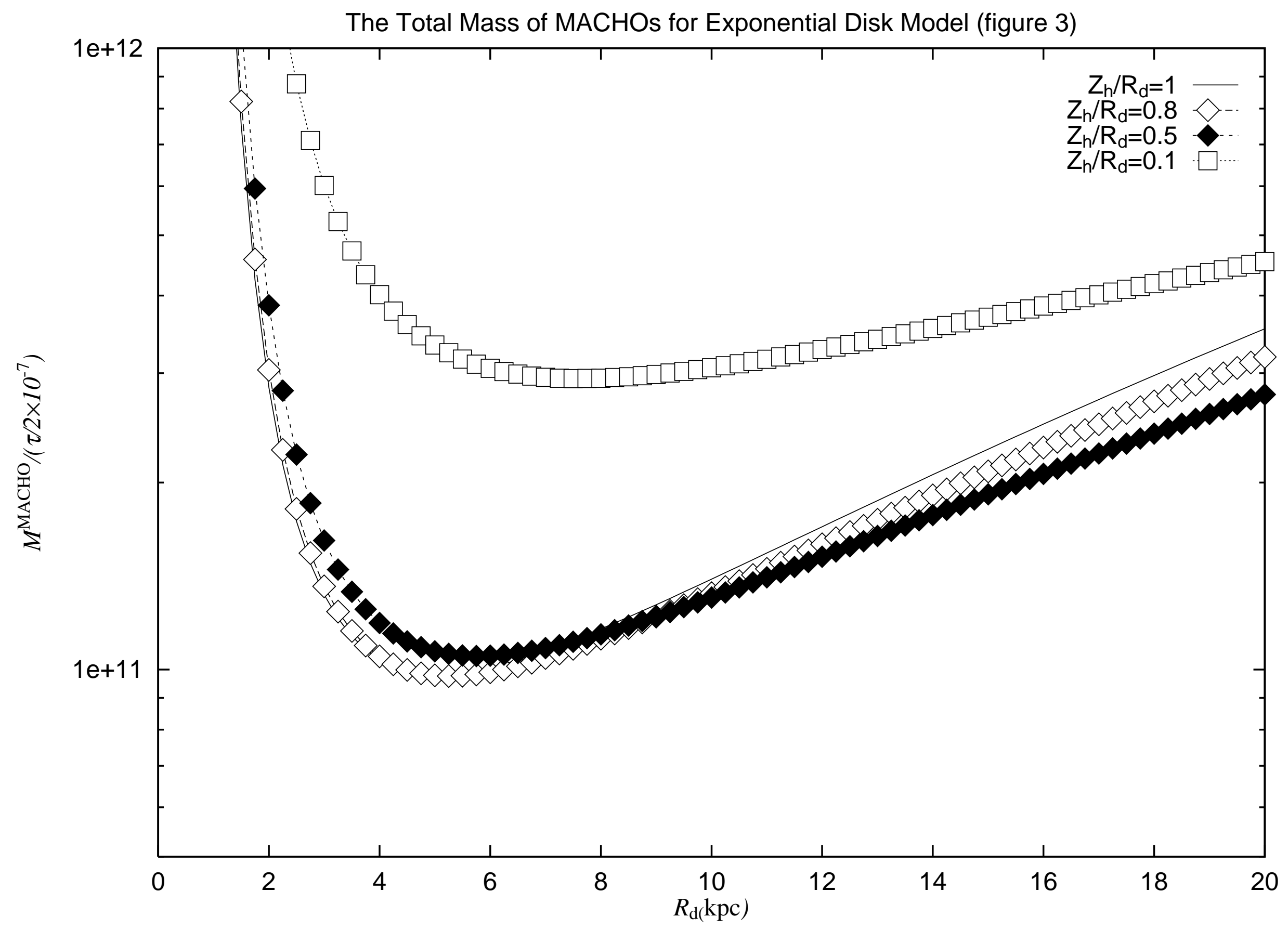


The Total Mass of MACHOs for Elliptic Model (figure 4a)

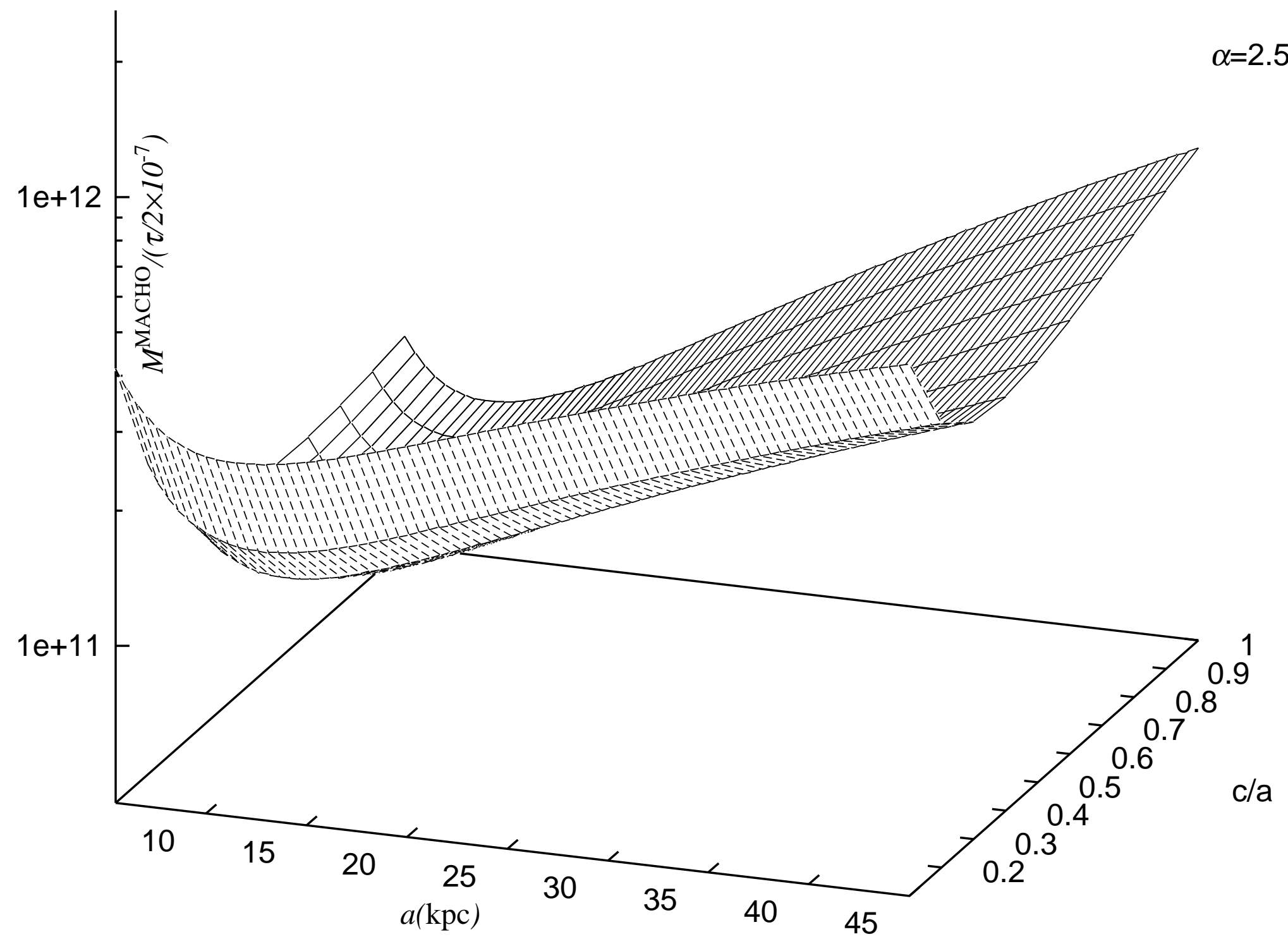




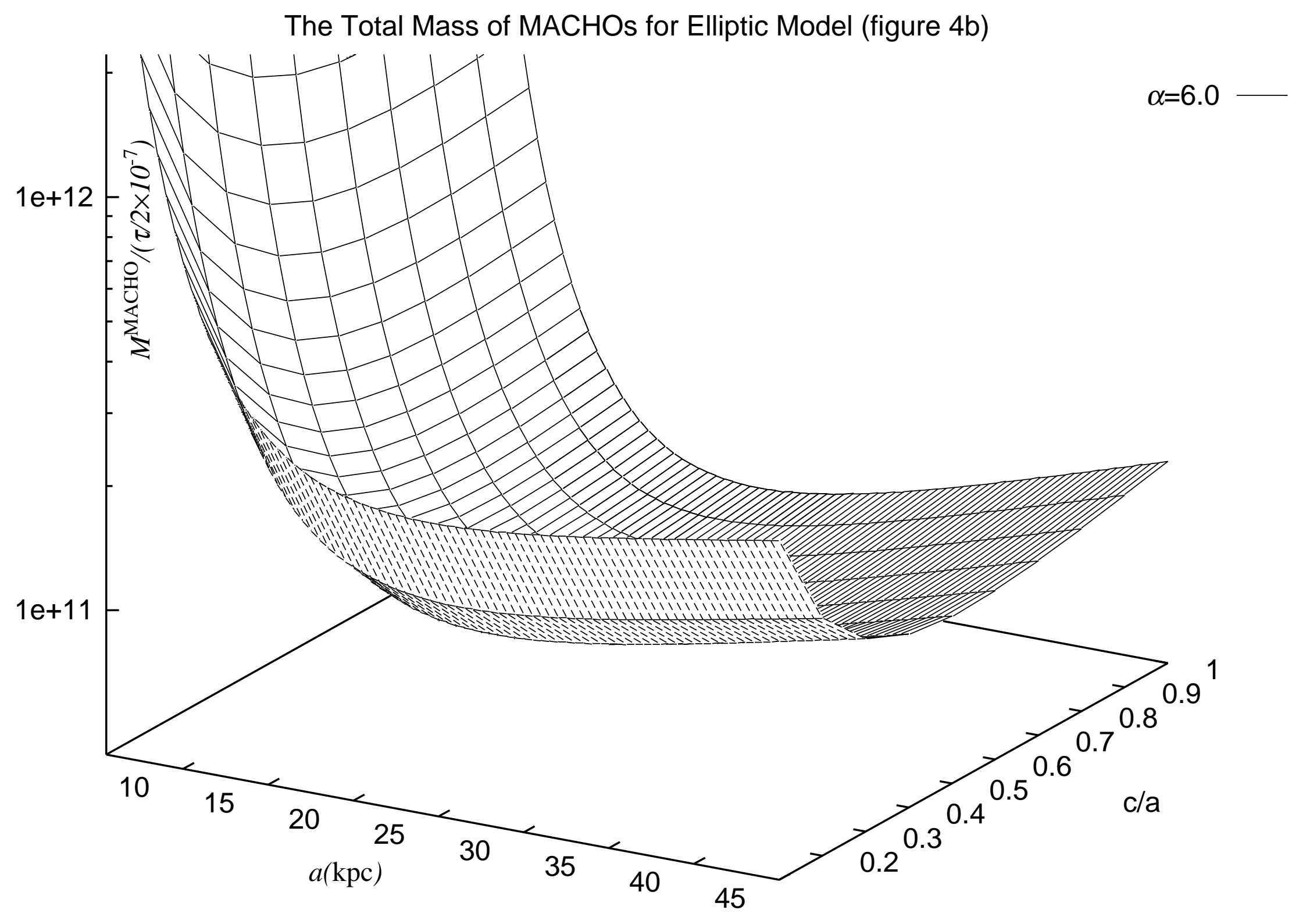

